NASA Technical Memorandum 101413

AIAA-89-0609

\title{
Evaluation of Three Turbulence Models for the Prediction of Steady and Unsteady Airloads
}

Jiunn-Chi Wu

Georgia Institute of Technology

Atlanta, Georgia

Dennis L. Huff

Lewis Research Center

Cleveland, Ohio

and

L.N. Sankar

Georgia Institute of Technology

Atlanta, Georgia

Prepared for the

27th Aerospace Sciences Meeting

sponsored by the American Institute of Aeronautics and Astronautics

Reno, Nevada, January 9-12, 1989 



\title{
EVALUATION OF THREE TURBULENCE MODELS FOR THE PREDICTION OF STEADY AND UNSTEADY AURLOADS
}

\author{
Jlunn-CW Wu* \\ Georgla Institue of Technc logy, Atlanta, GA 30332 \\ Dennis L Huff** \\ NASA Lewis Research Cenier, Cleveland, OH 44135 \\ LN. Sankar*** \\ Georgla Institute of Technclogy, Adlanta, GA 30332
}

\section{ABSTRACT}

Two-dimensional and quasl-3D Navler-Stokes solvers have been used to predict the statlc and dynamic airload characteristics of airfoils. The following three turbulence models were used: (1) BaldwinLomax algebraic model, (2)Johnson-King ODE model for maximum turbulent shear stress and (3) A two equation $k-\theta$ model with law-ofthe-wall boundary conditions. It was found that In attached fiow the three modeis gave good agreement with experimental data. In unsteady separated hows, these models gave only a falr correlation with experimental data.

\section{INTRODUCTION}

The flow field surrounding modern rotorcraft and propeller configurations is highly complex, and is dominated by threedimensional effects, transonic flow, flow separation and unsteadiness, and can be properly modeled only through the numerical solution of the 3-D unsteady Navier-Stokes equations. Since full 3-D simulations are costly, historically researchers have used simpler 3-D analyses such as the Iffting line theory, which use a table look up of 2-D steady and unsteady airfoil characteristics. The airfoll tables needed may come from carefully performed experiments, or from 2-D computer codes. To be uselul, the 2-D computer codes should provide:

1. Reliable prediction of airfoll statlc load data and dynamic stall characteristics.

2. A method for evaluation of the flow yaw effects on alload characteristics.

3. A suitable turbulence model for properly modelling separated flows.

In this study, 2-D and quash-3D computer codes have been developed capable of predicting the static and dynamic load characteristics of straight and swept wings. Three turbulence models are currently operatlonal. These are: (1) Baldwin-Lomax algebralc model, (2) Johnson-KIng ODE model and (3) Two-equation k-e model. This paper describes the performance of the above two computer codes for a variety of steady and unsteady flow conditlons. The effects of turbulence model on the predicted flow propertles are also evaluated.

\footnotetext{
- Graduate Research Assistant, Student Member AlAA. Presently, Associate Prolessor, National Central University, Talwan.

** Research Engineer, Member AIAA

*** Associate Professor, Member AIAA.
}

\section{MATHEMATICAL AND NUMERICAL FORMULATION}

In this work, the unsteady 2-D and quasl-3D, Reynolds inveraged, compressible Navler-Stokes equations are solved in a bodyfitted coordinate system using an alternating direction impliclt (ADI) procedure. The mathematical formulation has been described in detall It References 1 and 2, and only a brief outline of the formulation ts Given here.

\section{Soverning Equations}

The equations governing unsteady three-dimensional flow are the full Navier-Stokes equations, and may be written in a Carteslan coordinate system as:

$$
G_{t}+F_{x}+G_{y}+H_{z}=R_{x}+S_{y}+T_{z} \text { (1) }
$$

Here, $q$ is the unknown flow properties vector; $F, G$ and $H$ are the inviscid filux vectors; $R, S$ and $T$ are the viscous terms. The purpose of the present work is to compute the unsteady viscous flow over a bitrary configuratlons undergoing arbitrary motion. To facilitate this, th: 8 following general curvilinear coordinate system Is used:

$$
\begin{aligned}
\xi & =\xi(x, y, z, t) \\
\eta & =\eta(x, y, z, t) \\
\zeta & =\zeta(x, y, z, t) \\
& =1
\end{aligned}
$$

In such a coordinate system, equation (1) may be written in the following strong conservation form:

$$
q_{\gamma}+F_{\xi}+G_{\eta}+H_{\zeta}=R_{\xi}+S_{\eta}+T_{\zeta}
$$

The quantitles $\mathbf{q}, \mathbf{F}, \mathbf{G}, \mathbf{H}, \mathbf{R}, \mathbf{S}, \mathbf{T}$ are related to their Cartesian counterparts through the metrics of transformation.

In such a general coordinate system, the airfoll surface maps or to a 5 = Constant surface. The radlal direction is assoclated with the $\eta$ coordinate, and the direction normal to the airfoll maps onto $\xi=$ constant lines or planes. For a detalled description of the flow and fiux vectors in the cartesian and transformed coordinate systems, the re icier is referred to Ref. 1.

\section{Inlinite Sweep Assumption}

In many applications involving how over rotor blades, and propellers, the flow in reglons away from the root and the tip may be as sumed to be Invarlant along the spanwise direction. That is, a large spanwise or radial component of flow may exist, but the dertvathes of 
the flow properties along the radlal or spanwise direction may be assumed negligible. This assumption is sometimes called the "Inflinite sweep" assumption and is often used in the alrcraft industry to compute three-dimensional boundary layers, and to investigate their stability characteristics. Under the assumptions of inflinite sweep, the above equations become

$$
\mathbf{q}_{r}+F_{\xi}+H_{\zeta}=R_{\xi}+T_{\zeta}
$$

The equation set (4) conslsts of 5 equations, corresponding to the conservation of mass, energy, and $u, v, w$ momentum along the $x_{1}$, and $z$ directions respectlvely. In the special case where the blade sweep angle is zero, and the yaw angle of the flow relative to the blade is zero, the spanwise or radial component of velocity $v$ is identlcally zero, and the momentum equation along the $y$ - direction may be neglected, resulting in 4 equations.

\section{Solution Procedure}

The above equations are parabolic in time, and may be advanced in time using a suitable stable, dissipative scheme. In the present work, a formulation similar to that described by Steger [Ref. 2] was used. Standard second order accurate central differences were used to approximate the spatial derivatives, and to compute the metrics of transformation. The highly non-linear flux terms $F$ and $H$, which are unknown at a given time level ' $n$ ' were linearized about their values at a previous time level ' $n$ '. The time derivative was approximated as a first order accurate, two-point, backward difference. This leads to a system of simultaneous equations for the flow vector $q^{n+1}$. These equations were re-expressed as a pentadiagonal matrix system of simultaneous equations for the 'delta' quantity $\left(q^{n+1}-q^{n}\right)$. The penta-diagonal equation system was approximately factored into a product of tridiagonal matrices using the Beam-Warming approximate factorization scheme, as discussed in Ref. 1.

\section{Antificial Viscosity Mode}

The use of standard differences to approximate the spatlal derivatives can give ise to growth of high frequency errors in the numerical solution with time. To control this growth, a set of artificlal dissipation terms were added to the discretlzed equations. These dissipation terms used a combination of second and fourth order differences of the flow propertles, in a manner discussed by Jameson et al. [Rof. 3].

\section{TURBULENCE MODELS}

As stated earlier, three furbulence models were considered in this work. These models are briefly descried here.

\section{Baldwin-Lomax Mode}

This model is patterned after the well known Cebecl-Smith model, and has been extensively used by a number of researchers [Rel. 2,4$]$. It uses a two layer formulation to model the eddy viscosity. in the inner layer, the following expression is used.

$$
\mu_{t} \text { a } 1^{2}|(u y-v x)|
$$

where $I$ is the "mixing length" measured as the distance of the point from the nearest solid surface, modifled by the classlcal van Driest damping term, and the von Karman's constant. In the outer layer, the eddy viscosity is written as

$$
H_{1} \propto F_{\max } Y_{\max }
$$

where $F_{\max }$ and $Y_{\max }$ represent the turbulent velocity and length scales in the outer part of the boundary layer. The quantity $F_{\text {max }}$ is computed as the maximum of the following function:

$$
F(y)=y\left|\left(u_{y}-v_{x}\right)\right|\left[1-\exp \left(-y_{0} w_{w} w^{2} / 2 \omega_{w}\right)\right]
$$

where $y$ is the distance of the point from. the nearest solld wall. $Y_{\max }$ is the $y$ - location where $F(y)$ reaches a miximum. The quanthles $\rho, \tau$ and $\mu$ are the density, shear stress and viscosity respectlvely. The subscript $W$ represents conditions at the wall.

The Nebanofi intermittency factor is used to drive the eddy viscosity to zero far away from the boundary layer. In the wake regions downstream of the blade tralling edge. Baldwin-Lomax model is used with minor modifications. For a detalled discussion of this model, the reader is referred to Ref. 4.

\section{Jehnson-Klng One Equation Model}

The Baldwin-Lomax model is an equilibrium model in the sense that is assumes that the eddy viscosity instantaneously adjusts to the local flow characteristics. The Baldwin-Lomax model thus does not take into account the upstream eddy viscosity or turbulent kinetic energy values. Johnson-King model attempts to rectlfy this situation, by solving an ordinary differentlal equation (ODE) for the maximum turbulent kinetic energy within the boundary layer at a glven strearnwise location. This ODE may be thought of as a simplifled form of the Reynolds stress equation. This ODE is solved as an Intial value problem by marching along the how direction, and automatically brings into the account the upstream history of the flow.

The eddy viscosity $\mu_{t}$ is assumed to be

$$
\mu_{\mathrm{t}}=\mu_{\text {to }}\left[1-\exp \left(\mu_{\mathrm{t}} / \mu_{\mathrm{to}}\right)\right](\theta)
$$

where

$$
\mu_{\mathrm{q}}=0.4 \rho D^{2} y k_{m}
$$

and

$$
\mu_{\text {to }}=0.0168 \rho \sigma(x) u_{\theta} \delta^{*} \gamma(10)
$$

Here $D$ is the van Driest damping function, $y$ is the dlstance from the wall, and $k_{m}$ is the maximum Reynolds shear stress at the current $x$ - location. Furthermore, $y$ is the Kebanoff Intermittency factor, ue is the edge velocity, $\delta^{*}$ is the displacement thickness, and $\sigma(x)$ is a modelling parameter.

An ordinary differentlal equation is used to model the streamwise development of turbulent kinetic energy, and is glven by

$k_{m}^{1 / 2}=\left(k_{m}^{1 / 2}\right)_{\theta q}-\left[L_{m} u_{m} /\left(\varepsilon_{1} k_{m}\right)\right] d k_{m} / d x \cdot D_{m} L_{m} / k_{m}(11)$

The quantities $D_{m}, L_{m}, a_{1}$ are empirically prescribed as done in Reference 5. The subscript $m$ over some of the quantities indicates that these quantities are computed at the $y$-location where the local shear stress is maximum. The quantity $\left(k_{m}\right)$ is computed from equations 8,9 and 10 with the value of $\sigma(x)$ assumed unity. The quantity $\sigma(x)$ is iteratively determined, so that the value of the eddy viscosity computed from equations 8,9 and 10 and the maximum shear stress computed using equation (11) satisty the following relatlonship:

$$
k_{m}=\mu_{t m} / \rho\left|\left(u_{y}+v_{x}\right)\right|_{m}
$$

For an efficient iterative procedure for computing $\sigma(x)$, and the emplircal relationships used in the Johnson-King model, the reader is referred to Ref. 5.

\section{Gorskis ke Mode}

The third turbulence model considered in this work is the well known ke model, Implemented with a set of wall boundary conditlons proposed by Gorskl [Ref. 6]. Thls model requires numerical solution of 
two partial differentlal equations for the instantaneous values of turbulent kinetic energy $k$, and the dlssipation rate $c$ at every point in the flow field. These equations may be formally written as

$$
\begin{aligned}
& (6 k)_{q}+(\rho u k)_{x}+(6 v k)_{y}=\left(\mu_{k} k_{x}\right) x+\left(\mu_{k} k_{y}\right)_{y}+S_{1} \\
& \left.(\varphi \theta)_{t}+(\varphi v \theta)_{X}+6 v \theta\right)_{y}=\left(\mu_{\theta}^{e} x\right)_{X}+\left(\mu_{\theta} e y\right)_{y}+S_{2}
\end{aligned}
$$

Here $\mu_{\mathrm{K}}$ and $\mu_{\mathrm{e}}$ are eddy viscosities controlling the diffusion of $k$ and $\epsilon ; S_{1}$ and $S_{2}$ are source terms which describe the production and dissipation rates of $k$ and $\epsilon$. As in the case of the origlnal flow equations, these equations and their three-dimenslonal counterparts may be cast in a strong conservation form in a moving, body-fitted. curvilinear coordinate system. In the present work these equations were solved as a $2 \times 2$ system of partial differential equatlons using an $A O I$ procedure similar to that used to solve the mean flow equations. after the mean flow has been updated at a given time level.

in the vicinity of the solid wall, the values of $k$ and $\varepsilon$ computed from the above equations were overwritten with values computed from the following assumed relatlonships for $k$ and $\epsilon$ :

$$
\begin{aligned}
k & =C y^{2} \\
c & =\text { Constant }
\end{aligned}
$$

These constants were evaluated using the values of $k$ and computed at nodes well within the logarithmic reglon of the boundary layer, In a manner documented in detall by Gorskl [6].

\section{RESULTS AND DISCUSSIONS}

\section{Steady Flow Studies Using the Baldwin-Lomax Model}

A series of steady viscous calculations were performed using the Baldwin-Lomax model. In Figs. 1-5, a number of flow results are shown in the form of surface pressures, loads, skin friction and velocity profiles for a NACA 0012 airfoil and a supercritical RAE 2822 airfoil at a variety of flow conditions and compared with experimental data. The following flow conditions are considered:

Case 1 :Surface pressure distribution over NACA 0012 airfoll at free stream Mach number $M_{\infty}=0.301$, Angle of Attack $a=13.5$ degrees, Reynolds number based on chord, $R_{\theta}=3.9$ million.

Case 2: Static load characteristlcs for a NACA 0012 airfoll at $M_{\infty}$ $0.301, R \theta=3.9$ million.

Case 3: Predicted lift curve slope $d G_{1} / d a$ versus freestream Mach number for a NACAOO12 alrfoil at $a=0$ deg., Re $=9.0$ million.

Case 4: Surface pressure distribution over a RAE 2822 airfoil at corrected $M_{\infty}=0.73$, corrected $\alpha=2.79$ deg., $R_{\theta}=6.5$ Million.

Case 5: Skin friction coefficlent and velocity profiles on the upper airfoil surface for a RAE 2822 alroll at $M_{\infty}=0.73, \alpha=2.79$ deg., $R \theta=6.5$ million.

As seen In Flg. 1, good agreement was found between the computed surface pressures and experiment [7] at a high angle of attack flow prior to statlc stall. The static load characteristics of the NACA 0012 airfoil shown in Fig. 2 indicate good prediction of static lih and moment stall. However, the drag was overpredicted at high angles of attack. In these calculations, the transitlon effects on loads prediction were simulated by enforcling the transition point at $0.05 \%$ chord on both the upper and lower surfaces. In Fig. 3, a good agreement for the litt curve slope is observed between the present predictions and the experiment [8]. For the super-critical RAE 2822 airfoil it is found that the predicted surface pressures shown on figure 4 are in good agreement with the experiment [9] over most of the airfoil. The predicted pressure expansion near the leading edge on the upper airfoil surface is not as strong as observed in the axperiment, presumably because the flow was assumed to be urbulent over the entire alifoll surface in the present calculations. The integrated ift and drag from the experiment are 0.7433 and 0.0127 . respectively, which compare well with the values of 0.7432 and 0.0134 from the present code. Fig. 5 shows that the skin friction in predicted well except near the trailing edge. The computed veloclty profles at iwo chordwise locations are in good agreement with the experiment.

Additional viscous flow calculations computed using this solver may be found in Ref. 1.

Jynamic Stall of Oscillating Alriolls Comouted Usine Baldwin-Lomax liodel

A number of dynamic stall calculatlons have been performed for several 2-D aifoils and a swept wing configuration with infinitely ling span. The airfoils analyzed in the 2-D mode are a NACA 0012 airfoll and two modem helicopter airfoll sectlons (the SIkorsky SC-1095 airfoll and the Hughes HH-02 Airfoil). The 2-D dynamic stall results are presented in Figs. 6 Through 13. The angle of attack varlatlon during the dynamic stall is given by $\alpha=15-10 \cos (\omega t)$. The free stream N'ach number In these three cases was 0.28 , the Reynolds number based on chord was 3.7 Million, and the reduced frequency based on 8:mi-chord was 0.15 .

The calculations were carried out for two cycles of alfoll pitching motion starting with a steady state solution at 5 degree angle of attack, to remove influence of fiow transients. Here results for the sicond cycle are shown. In figure 6, the dynamic stall calculations are presented lor two grids: a $157 \times 58$ grid and a $253 \times 58$ grld.

As can be seen In Fig. 6, predicted loads using the coarse grid a jree wh experiments [7] reasonably well during the upstroke. Aithough the maximum lift coefficlent is underpredicted and moment prediction was less accurate, the theory still clearly captures the moment and lift stall. The leading edge separation occurred when thie angle of attack is 17 degree during the upstroke. A large "primary" vortex formed subsequently. As the alfoll Incidence increased, this primary vortex passed along the airfoil surface and was shed into the wiake at an angle of attack around 25 degrees. During the downstroke, hiwever, the egreement between the numerical results and experimental data is not good. A secondary vortex from the trailing exige was shed during the downstroke. It appears that the strength of the secondary vortex is overpredicted (which takes place at a between 25: to 23 degrees). As the incidence of the airfoil continues to dicrease, the agreement between predicted and measured loads inproves.

The dynamic stall predictions for the Hughes $\mathrm{HH}-02$ alrfoll and this Sikorsky SC-1095 airtoil are shown In Figs. 7 and B. Good agreement between the theory and experiment is also observed during this upstroke for these two cases. During the downstroke, the computed results are only in falr agreement with the experimental data. Overall levei of agreement for these two airfoils is simllar to that for the NACA 0012 airfoil.

In order to evaluate the effects of yaw angle on statlc and dy namic load characteristlcs, a series of calculations which take into acicount the effect of the blade sweep relative to the treestream, and thus the effect of radial flow on the load characteristics, have been done. In Fig. 9, the static if versus angle of attack are shown for a NACA 0012 alrfoil at 0.3 Mach number and $R e=2.7$ million at 30 degree yaw angle. Comparisons with the experimental data of Carta [11] are also given. Good agreement between the two sets of data is obsierved.

In Figs. 10 and 11, the dynamic stall load characteristics of a NACA 0012 aifoil at 0 and 30 yaw angle are compared. The flow conditions are: $M_{\infty}=0.3, R e=2.7$ million. For comparison, Carta's results are also given. Only a qualitative agreement between the two sels of data is observed. The yaw effects on dynamic stall hysteresis locps are predicted to be less prolound than observed in experiment. Several factors may contribute to the discrepancies. Firstly, the 
location of transition is somewhat different between the two sets of data. In the computation, the flow is assumed to be fully turbulent over the entire alroil. In Carta's experiments transitlon depended on factors such as surface roughness, mean fiow turbulence level, etc. and is difficult to model. Secondly, three-dimenstonal effects of the swept wing with finite span considered In the experiment may be important and the present quasi threo-dimensional approxlmation may not suitable for this conflguration.

\section{TURBULENCE MODEL STUDIES}

In order 10 assess the effects of turbulence models on the prediction of separated flows, a number of turbulent flow solutions have been computed using the three turbulence models and have been compared with each other and also with avallable experimental data. Several steady flow situations were considered. These are: turbulent boundary layer over a flat plate; attached subsonic flow over airfoil; and separated transonlc flow over an aiffoil. Following these studies, calculations were made for an airfoll experlencing dynamic stall, using higher order turbulence models.

\section{Artached Flows}

First, some results are presented for a turbulent boundary layer developing over a flat plate at zero angle of attack. The flow is nearty Incompressible and the Reynolds number is five million [11]. The calculations were performed on a computational reglon which extended half plate length upstream and downstream of the flat plate. and one plate length above the plate surface; 151 equally spacing nodes were used along the streamwise and 51 nodes were placed at geometrically increasing distance in the normal direction. The first point off the surface located within a $y+$ of 2 . The predicted surface skin friction and velocity profile at mid-plate are shown In Fig. 12 for the three models. These three models predlct results that compare very well with experiment. Note that velocity characteristics of the sublayer and buffer reglons were correctly captured by these models. This resolution is crucial to the success of turbulence model in resolving the near-wall turbulent flow characteristics.

Next results are presented for a turbulent attached flow pas an airfoil. Fig. 13 shows computed and experimental pressure distributions for the NACA 0012 alrfoil at an attached flow conditlon $\left(M_{0}=0.301, \alpha=13.5\right.$ degrees and $R_{\theta}=3.9$ million). The computed surface pressures are In good agreement with experimental data [7] It should be mentloned that the Johnson-KIng model which requires solution of an ODE starting from a user-prescribed point has some uncertainty as to where it should be activated. The activating location should be a finite dlstance downstream of stagnation point or transition location. Several locatlons were tested $(x / c$ ranging from 0.08 to 0.25 ) and it was found the solution in this case was not aftected by the starting location.

Transonic Flows with Separation

Two transonic flow cases exhlbhing mild and strong separation have been computed and compared with detalled turbulent flow measurements. In Fig. 14, the surface pressures are shown for a NACA 0012 airfoil at experimental conditions $[8]: M_{\infty}=0.899$, Angle of attack $=2.66 \mathrm{deg} . \mathrm{Re}=9 \mathrm{M}$ Million. It is seen that the Baldwin-Lomax and the $k$ - models predict similar pressure distributions, with a shock predicted stronger than the measurements. The predicted pressure distribution using the Johnson-King model is dependent on where the non-equilibrlum formulation is activated. The location $x / c=0.15$ gives best agreement with measured surface pressures. However, it is not clear how a starting location may be chosen.

The NACA 64A010 airoil at a shock induced stall condition [12] ( $M_{e}=0.8, \alpha=6.2 \mathrm{deg}$. $R_{\theta}=2$ Million) has also been considered for detailed turbulent flow comparisons. As can be seen in Fig. 15, the predicted pressure distributions are not in good agreement with experiment for any of the models. All of them predicted too strong and aft a shock and too little pressure recovery. It was found that the Johnson-King model the activation location of the nonequilibrium calculation was chosen at $x / c=0.15$ ) predicted a shock location forward of the other models. It should be mentioned that all these models showed some unsteadiness $(5 \%)$ In their loads predictions (buffeling). Mean veloctty and Reynolds shear stress proflles for thls case are compared in Fig. 16. Except In the close-to the-wall and near-wake regions, reasonable agreement whe the measured mean-velocity proflles is found for all the three models. Because of the thlck boundary layer predicted and the underprediction of pressure recovery, the predlcted mean velocity profiles do not match well with experimental data close to the wall and this effect also extends to the near-wake region $(x / c=1.02)$. Poor predictions are lound for Reynolds shear stress proflles for all of the models. The shear stress peaks are underpredicted and their locations shift more closer to the wall compared to experimental date.

The behavior of the $k-$ model is somewhat similar to the Baldwin-Lomax algebralc modet In this case. However, a thinner reversed flow is observed using the $k-$ model. Additionally, the $k-t$ model shows a better prediction of the Reynolds shear stress profiles close to the wall.

From the two transonic flow cases just described, it appears that the $k_{-}$model does not hold any noticeable advantage over the simple Baldwin-Lomax model in predicting the shock-Induced separated flows. It has been pointed out by Lakshminarayana [13] that the $k-$ model does not predict the separation point or the reattachment any more accurately. Therefore, inaccurate predictions of velocity and Reynolds shear stress profiles in the separated regions were not surprising.

As for the Johnson-King model, it is seen that the solutions depend on the choice of activation location of the non-equilibrium calculation at least in separated flows. Thls shows that the optimum locatlon differs from flow to flow. For unsteady. separated flow such as the dynamic stall problem, it would be difficult to determine the optlmum location during the osclllating airfoil motion. In addltion, calculations using the Johnson-King model tend to show small oscillations about mean loads lor the steady cases and require more Iterations than other two models to ensure convergence. Thus, it is concluded that the Johnson-Klng model Is not sultable for predicting the unsteady, highly separated flows. In dynamic stall calculations to be discussed next, only the Baldwin-Lomax algebralc model and $k-\varepsilon$ equatlon model were used and compared.

\section{Unsteady. Highly Separated Flow (Dynamic Stall Case)}

The dynamle stall calculation for NACA 0012 airfoil previously reported was repeated for an comparison of the $k-$ and the BaldwinLomax models for thls complex flow. Predicted aerodynamic loads are presented in Fig. 17 and compared with experiment [7]. The kt model predicts higher litt during the upstroke. During the downstroke, predictlons using the k-e model show trends similar to the BaldwinLomax model, except that a smaller second vortex shedding (around 24 deg.) and much stronger third vortex-shedding (around 15 deg.) were detected. Results from both models only show a qualitative agreement with the experiment during the downstroke.

\section{COMPUTER TIME REQUIREMENTS}

The calculations presented in thls work were performed on a CRAY X/MP supercomputer at the NASA Lewis Research Center. The computer time for a viscous solutlon using the Baldwin-Lomax model is 0.28 second per time step using a $157 \times 58$ grid. A converged viscous steady solution requires 2000 to 3000 time steps when a space-varying time step technlque is employed (roughly equal to 850 seconds). For dynamic stall cases, the computer time required for a full cycle on a $157 \times 58$ and a $253 \times 58$ grid was 4100 seconds and 6600 seconds, respectively. The swept wing configuration, requires $20 \%$ more computing time than the 2-D version, because an additional (spanwise momentum) equation needs to be solved. Computer time for the Johnson-KIng modet is about 0.30 seconds per time step, and requires about 500 to 1000 more time steps than the Baldwin-Lomax model to achieve a steady state. For the $k-t$ model, one time step requires 0.34 seconds and the same number of time steps as the algebralc Baldwin-Lomax model are needed to achieve a steady solution. 


\section{CONCLUSIONS}

An efflcient solution procedure has been used to provide improved prediction of complex flow phenomena assoclated with rotor flows. The two-dimensional, and quasl-three dimensional, compressible, full Navier-Stokes equations have been solved using an ADI scheme. Numerical results show that good prediction of static loads and dynamic stall hysteresis loops of rotor blade sectlons was feasible. Evaluation of three eddy viscosity models have been made. In attached flows the three turbulence models considered gave good correlation with experimental data. For strongly separated flows, eddy viscosity models avallable including the $k-$ model are not adequale. No clear trend could be found favoring the use of higher order turbulence models in separated flows.

\section{ACKNOWLEDGEMENTS}

The turbulence modeling work was supported by the NASA Lewis Research Center under Grant No. NAG 3-768. Studies related to the effect of yaw angle were supported by a grant from the McDonnell Douglas Helicopter Company. The authors wish to thank Dr. LS. King of the NASA Ames Research Center for providing the Johnson-King turbulence model subroutine.

\section{REFERENCES}

1. Wu, Jiunn-Chl., "A Study of Unsteady Turbulent Flow past Airfolis," Ph. D. Thesis, Georgia Institute of Technology, June 1988.

2. Steger, J. L." Implictt Finite Difference SImulation of Flow about Arbitrary Two-Dimensional Geometries, "AlAA Journal, Vol. 18, No. 7 July, 1978, pp. 679-686.

3. Jameson, A., Schmidt, W. and Turkel, E., "Numerical Solutions of the Euler Equatlons by Finite Volume Methods Using Runge-Kutta Time Stepping Schemes," AlAA Paper 81-1259.

4. Baldwin, B.S. and Lomax H., Thin Layer Approximation and Algebralc Model for Separated Turbulent Flows, AIAA Paper 78-0257.

5. Johnson, D.A. and King, LS., "A Mathematically Simple Turbulence Closure Model for Attached and Separated Turbulent Boundary Layers," AIAA Journal, Vol. 23, No. 11, Nov. 1985, pp. 1684-1692.

6. Gorskl, J.J. "A New Near-Wall Formulation for the k-e Equations of Turbulence," AIAA Paper 86-0556.

7. McAlister, K.W., Puccl, S.L. McCroskey, W. L and Carr, LW., "An Experimental Study of Dynamic Stall In Advanced Alroll Sections, Vol 2. Pressure and Force Data," NASA TM 84245, Sept, 1982.

B. Harris, C.D., Two-DImensional Aerodynamic Characteristics of the NACA 0012 Airfoil in the Langley 8-foot Transonic Pressure Tunnel," NASA TM-81927, 1981.

9. Cook, P.H. McDonald, M.A. and Firmin, M.C.P., "Aerololl RAE 2822 Pressure Distributions, and Boundary Layer and Wake Measurements, "AGARD Advisory Report No. 138, May 1979.

10. St. Hilaire, A.O. and Carta. F.O.. 'Analysis of Unswept and Swept Wing Chordwise Pressure Data From an Oscillating NACA 0012 Airfoil Experiment," NASA CA 3567, Vol I-Technical Report, March 1983.

11. Welghardt, $K$. and Tillmann, $W$. ., On the Turbulent Friction Layer for Rising Pressure," NACA TM 1314, 1951.

12. Johnson, D.A. and Bachalo, W.D., Transonlc Flow past a Symmetrical Airfoil-inviscid and Turbulent Flow Propertles," AIAA Journal, Vol. 18, Jan. 1980, pp. 16-24.

13. Lakshminarayana, B., "Turbulence Modeling for Complex Flows," AIAA Paper 85-1653, 1985.

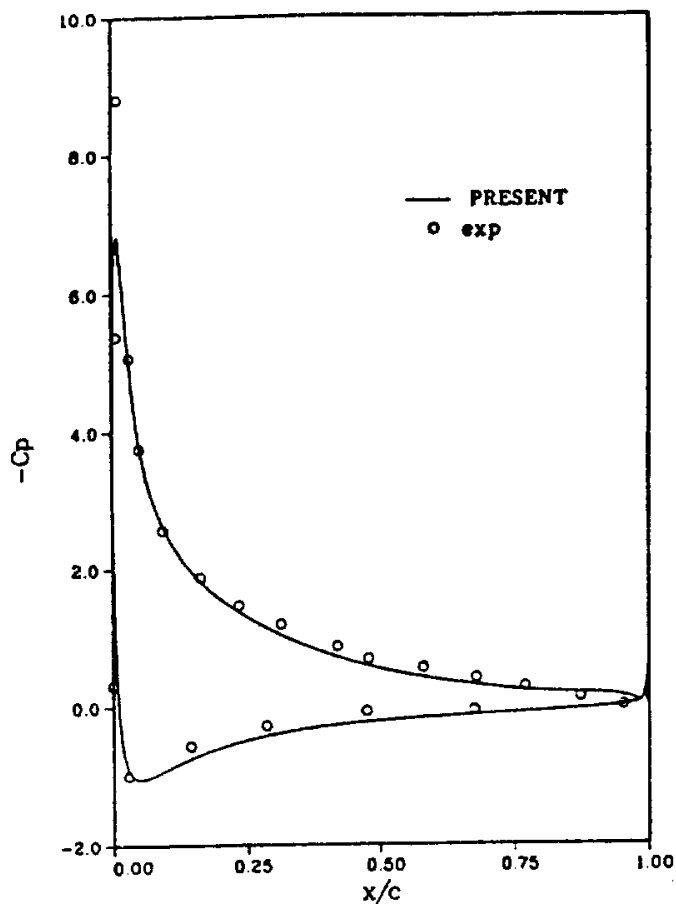

1. Surface Pressure Distribution over a NACA 0012 Alrioll at $M_{6}=$ $0.301, \alpha=13.5$ degrees, Reynolds Number = 3.9 Million.
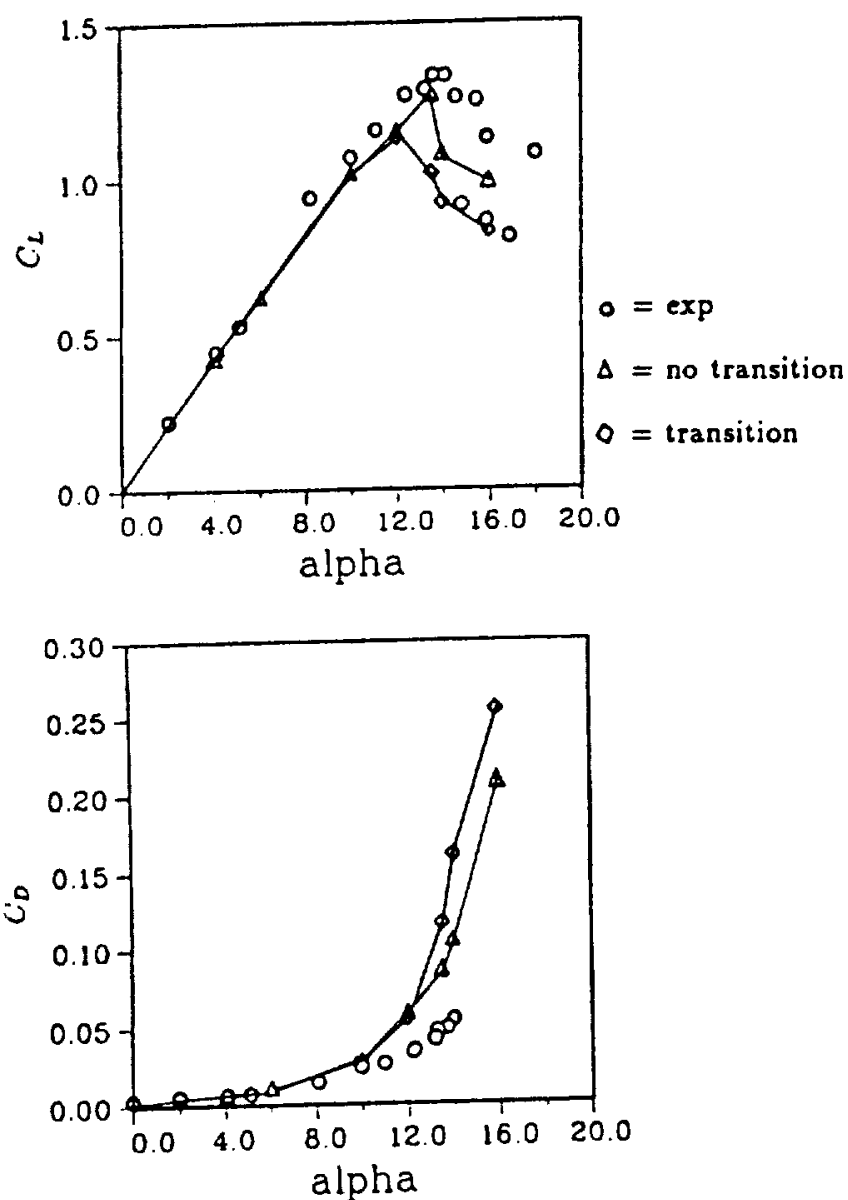

2 Computed and Measured Statlc Loads over a NACA 0012 Airfoil at $A_{\infty}=0.3, R_{\theta}=3.9$ Millon. 


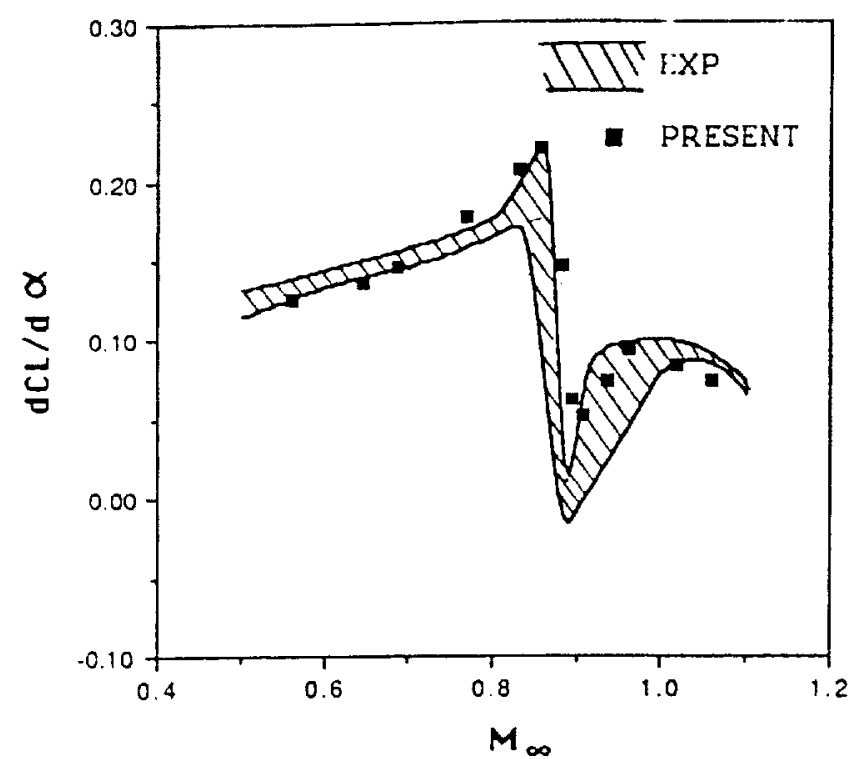

3. Predicted and Measured Lift Curve Slope vs. Mach Number for a NACA 0012 Airfoil at $\alpha=0$ degrees, $R \theta=9$ Million.
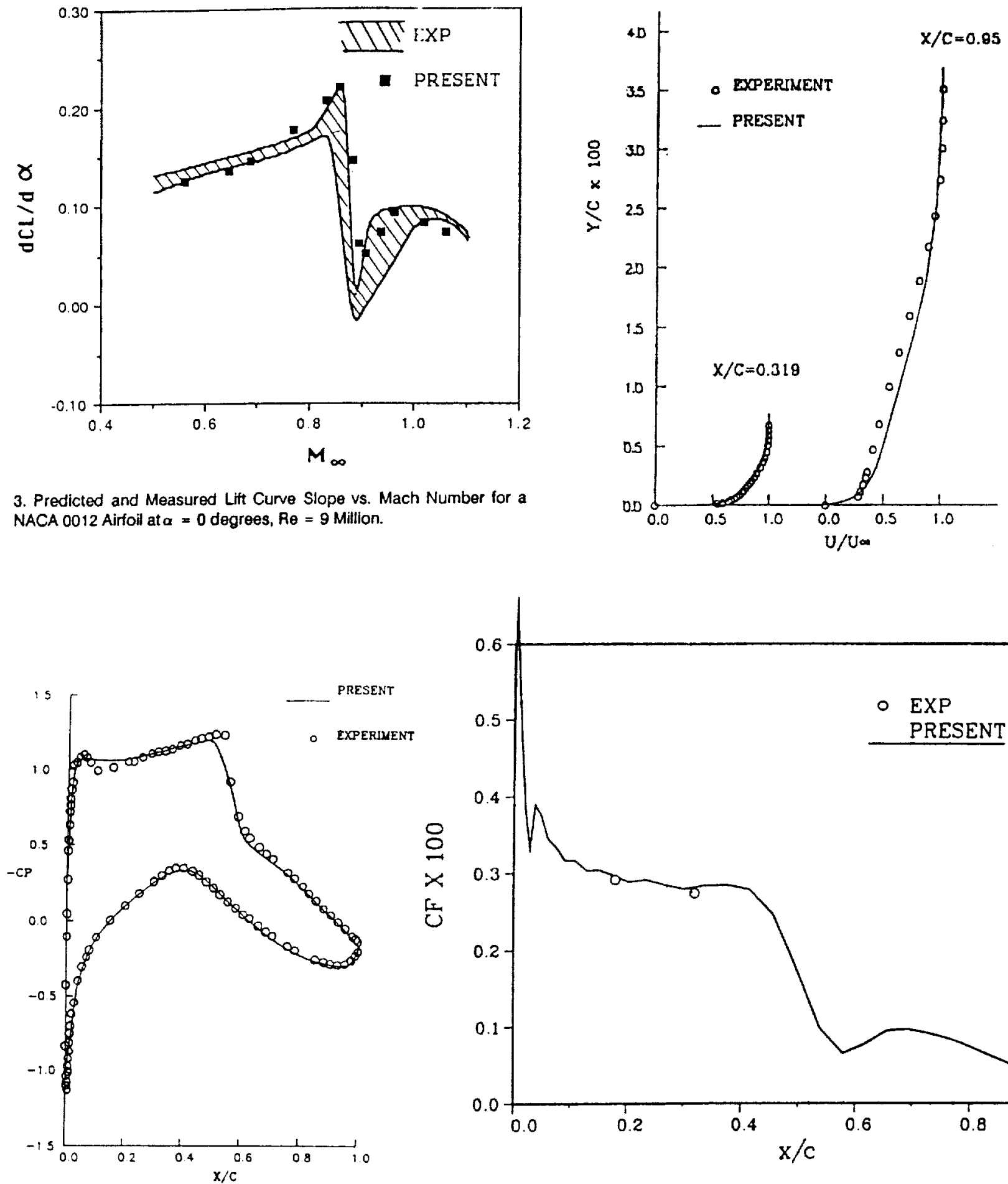

4. Surface Pressure Distribution over a RAE 2822 Airfoll at $M_{\infty}=0.725$, $\alpha=2.92$ degrees, $R_{\theta}=6.5$ Million
5. Velocity Profiles and Skln Friction Distribution for Conditions shown on Figure 4. 


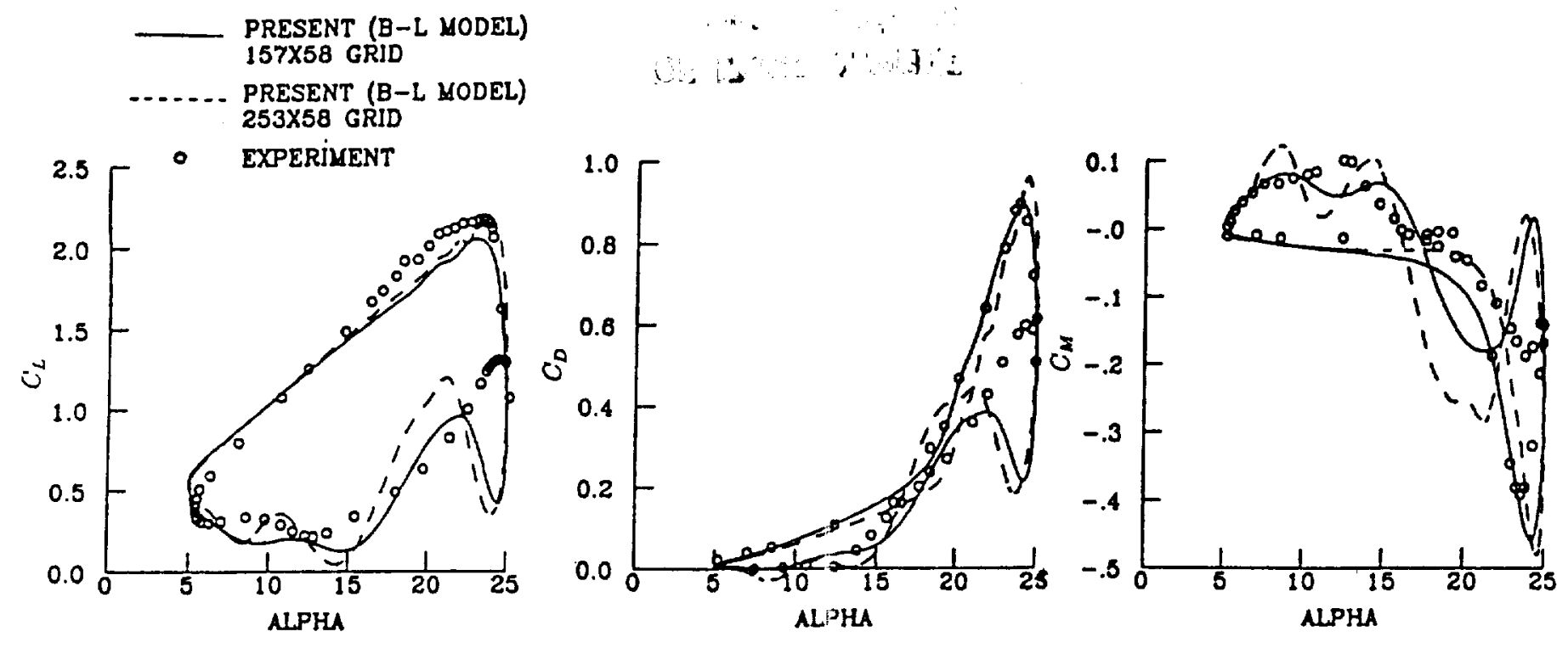

6. Computed and Measured Loads for a NACA 0012 Alfoll at Deep Dynamic Stall Condtion. $M_{6}=0.283, F_{\theta}=3.45$ Malion, Reduced Frequency 0.151.
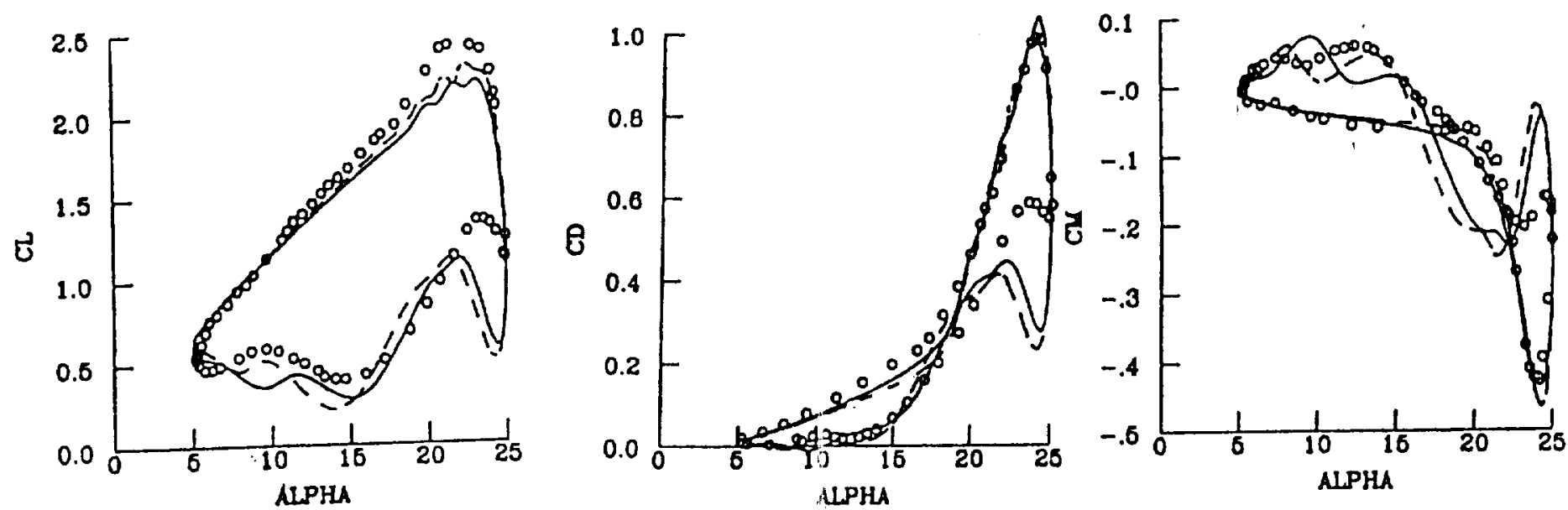

7. Computed and Measured Loads for a Hughes HH-02 Alfoll at Deep Dynamic Stall Condition. $M_{\infty}=0.283, R_{e}=3.45$ Million, Reduced Frequency 0.151.
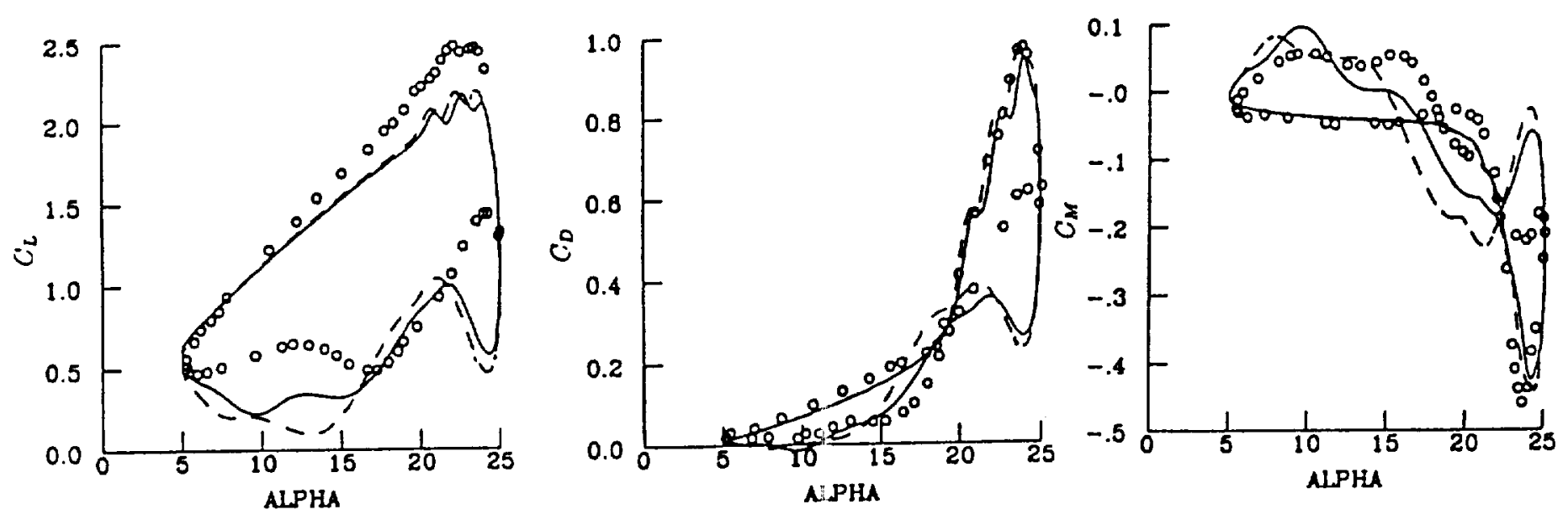

8. Computed and Measured Loads for a SC-1095 Airfoll at Deep Dynamic Stall Condition. $M_{6}=0.283, R_{\theta}=3.45$ Million, Reduced Frequency 0.151 . 


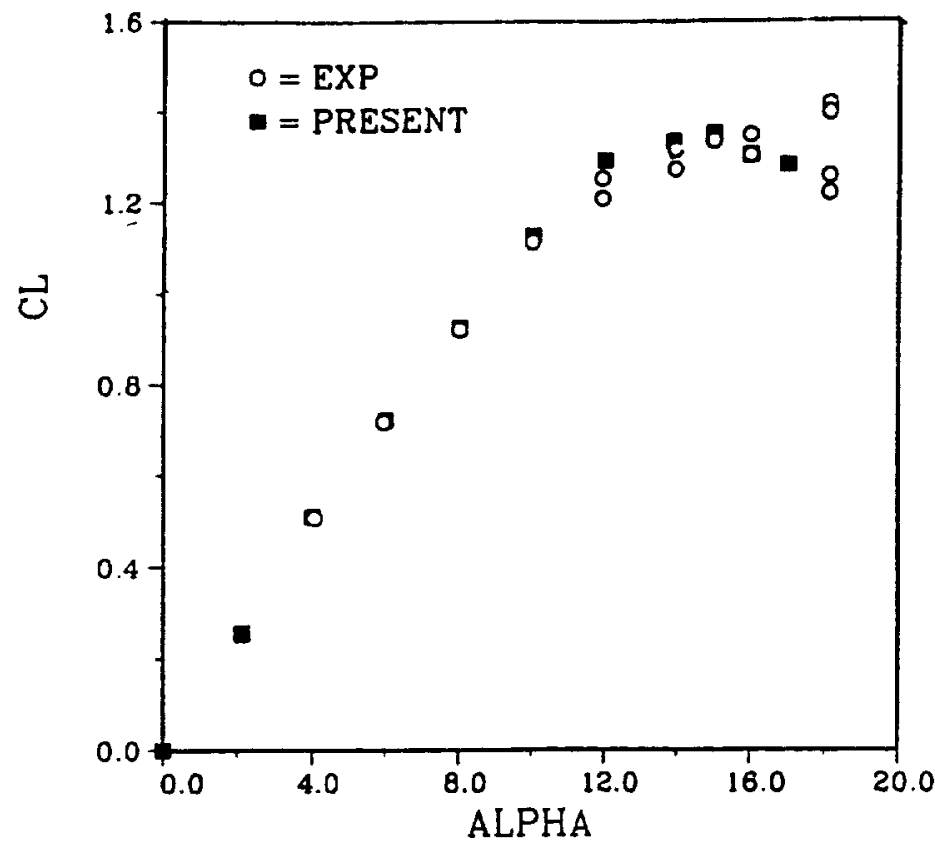

ORIGINAL PAGE IE
OE POOR QUALITY

9. Litt vs. Angle of Attack over a NACA 0012 Alfoll at 30 degree yaw. Chordwise Mach Number 0.346, Re = 2.77 Million.
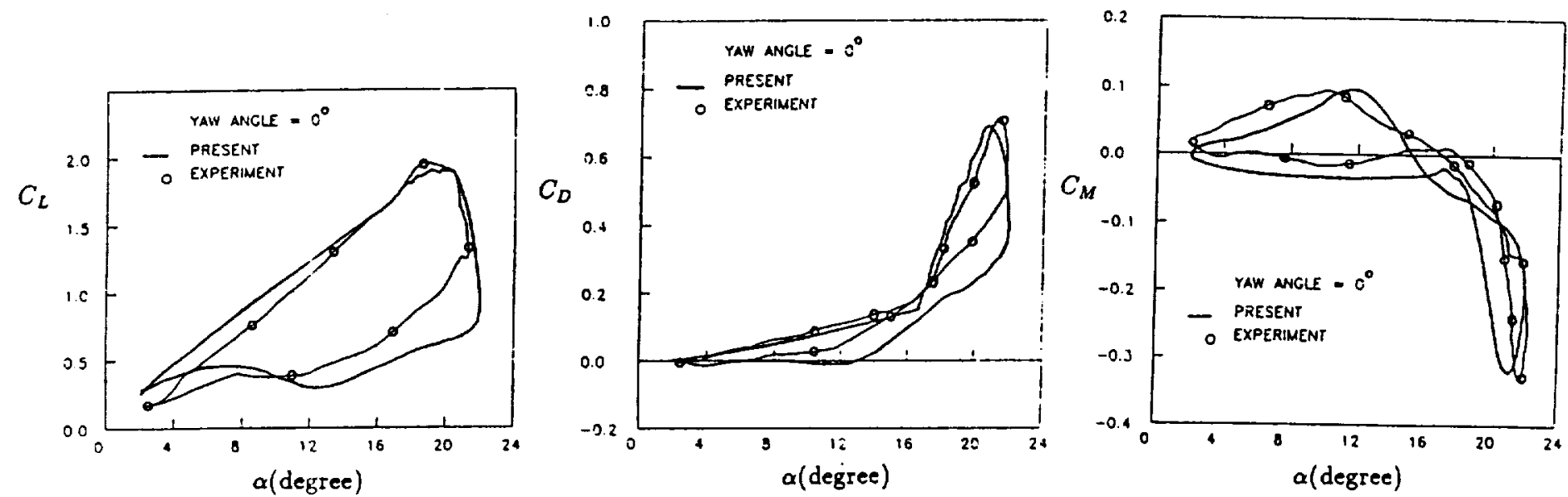

10. Computed and Measured Loads for a NACA 0012 Airfoil at Deep Dynamlc Stall Condition. $M_{6}=0.3, R_{\theta}=2.77$ Milion, Reduced Frequency 0.124 , Zero Yaw.
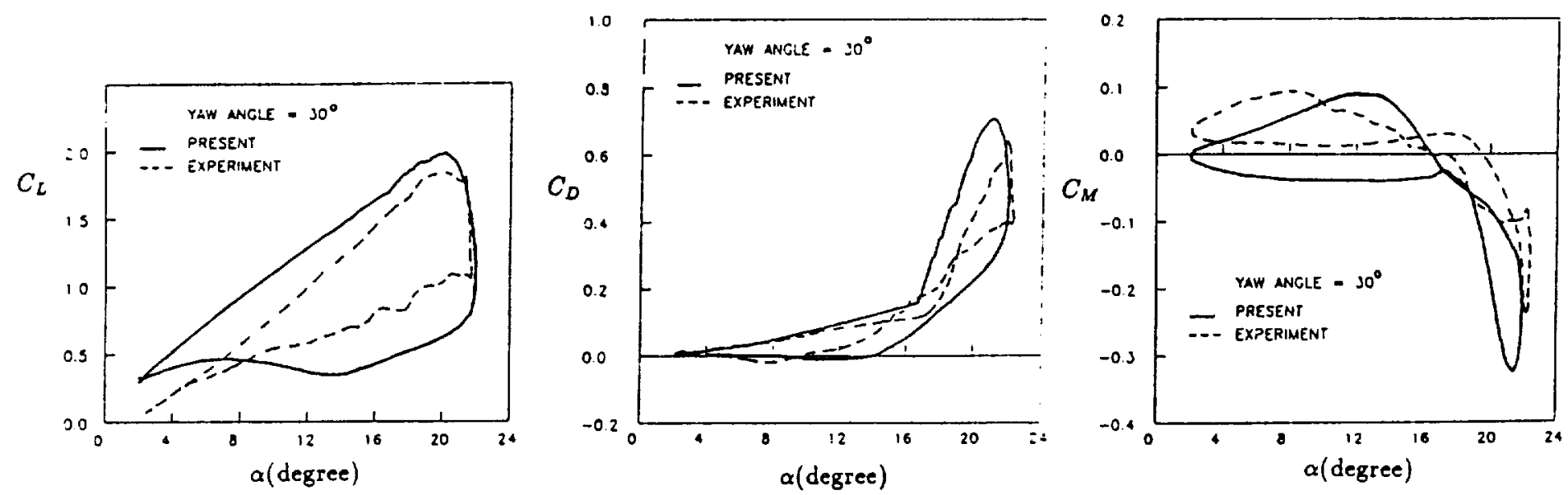

11. Computed and Measured Loads tor a NACA 0012 Airfoil at Deep Dynamic Stall Condition. $M_{0}=0.3$, Re $=2.77$ Million, Peduced Frequency $0.124,30$ degree Yaw. 

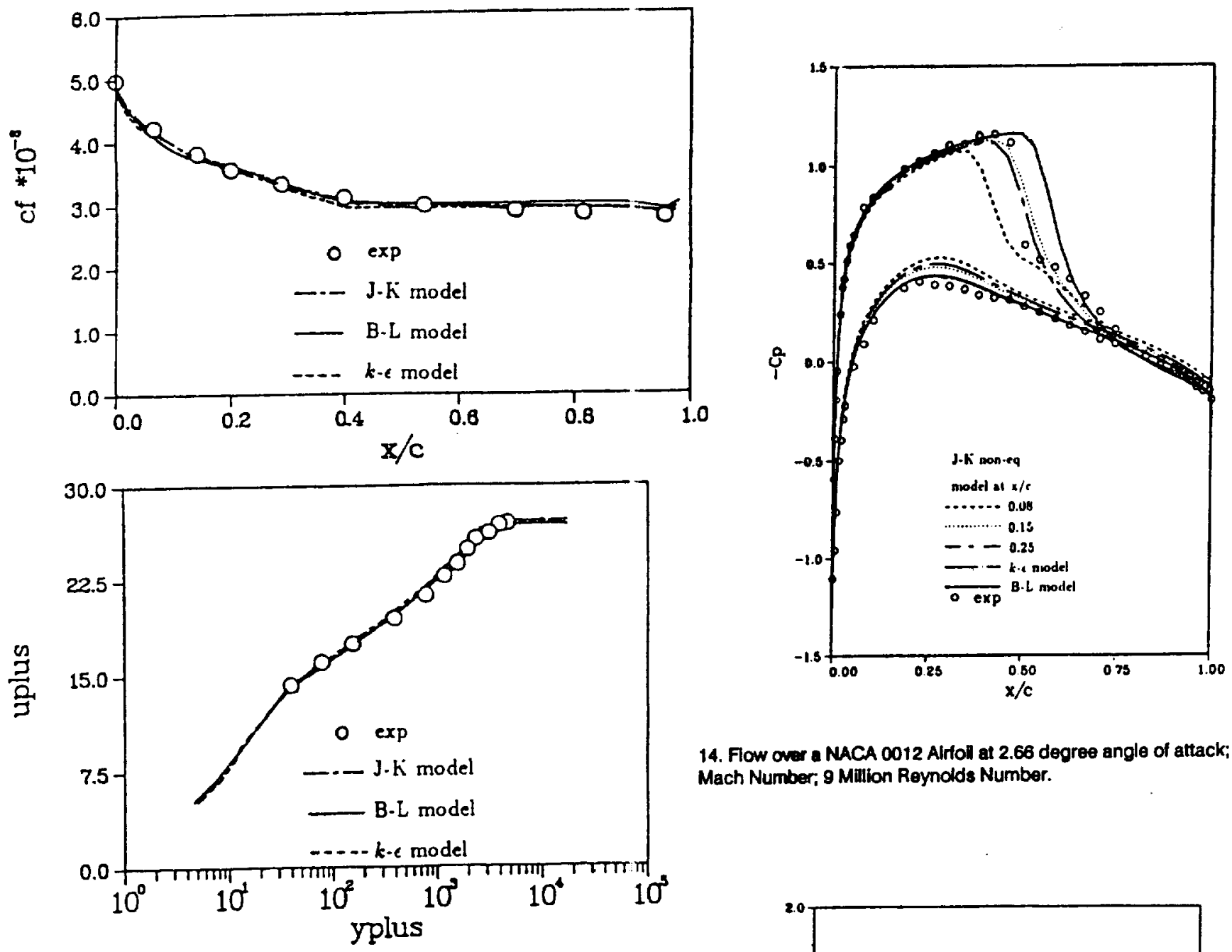

12. Surface Skin Friction and Velocity Profile Comparisons for a Flow over a Flat Plate. $M_{\infty}=0.1, R_{\theta}=5$ Million.

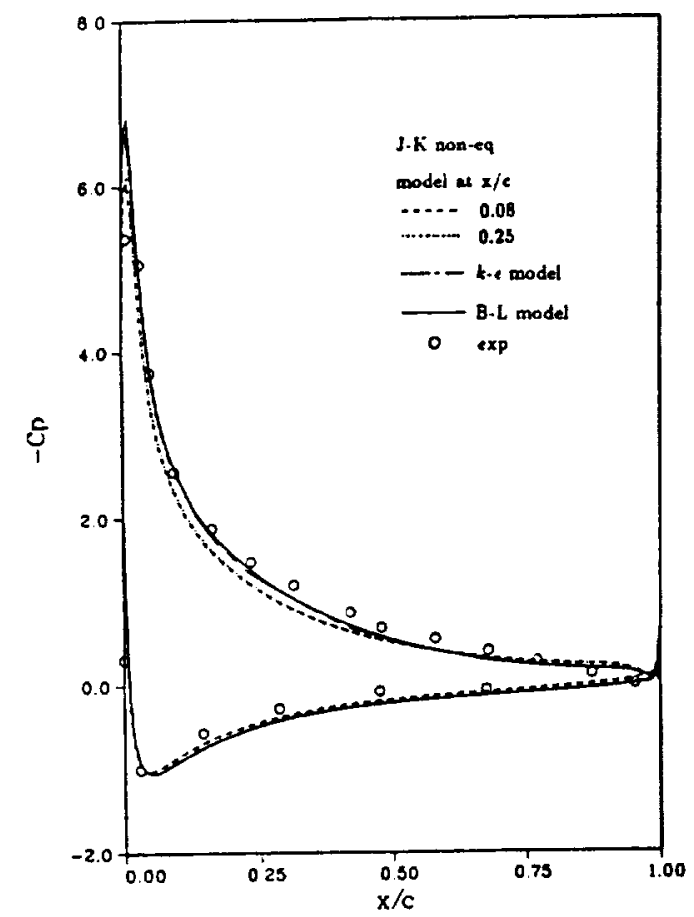

13. Flow over a NACA 0012 Alfoll at 13.5 degree angle of attack; 0.3 Mach Number; 3.9 Million Reynolds Number.

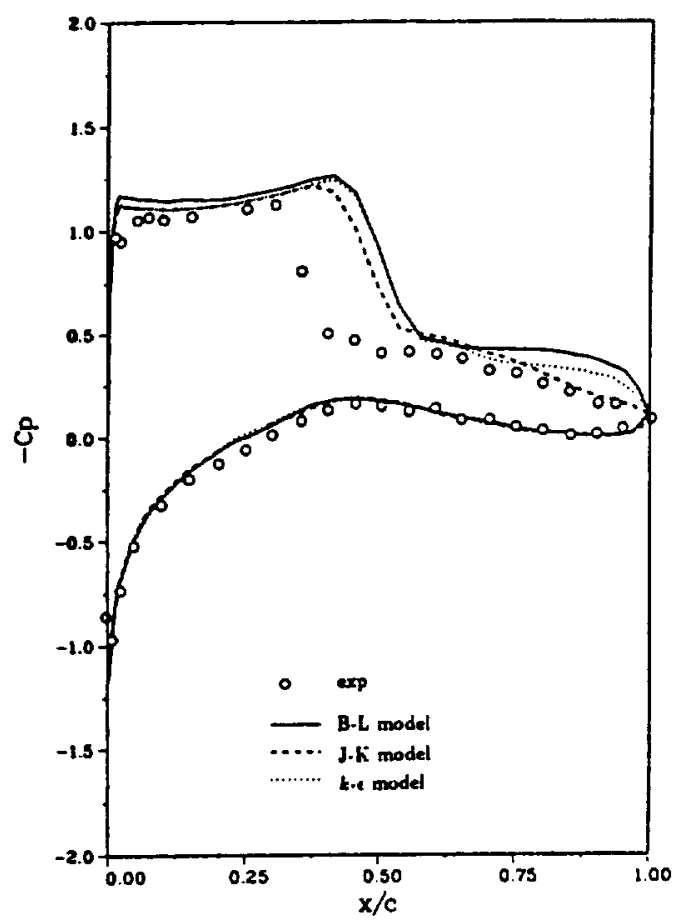

15. Flow over a NACA $64 A 010$ Alrfoll at 6.2 degree angle of attack; 0.8 Mach Number, 2 Millon Reynolds Number.

$$
\text { Prgan: }
$$

14. Flow over a NACA 0012 Airtoll at 2.66 degree angle of attack; 0.799 Mach Number, 9 Malion Reynolds Number. 
$0 \exp$

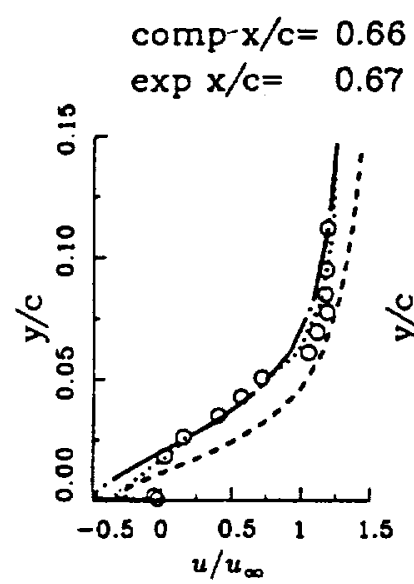

$$
\operatorname{comp} x / c=0.82
$$

$\exp \mathrm{x} / \mathrm{c}=0.83$

comp $x / c=1.02$
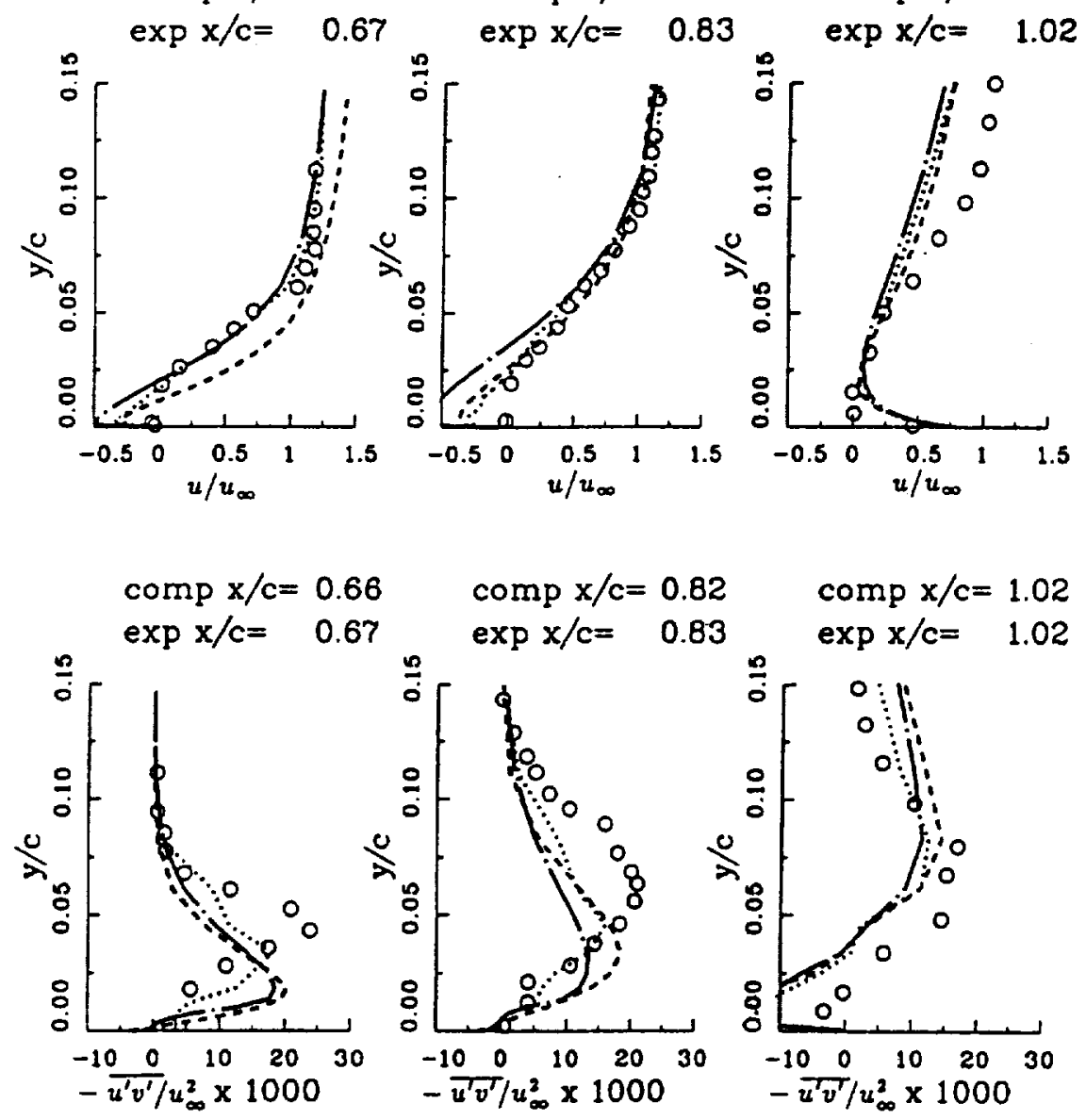

$\operatorname{comp} x / c=0.82$

$\operatorname{comp} x / c=1.02$

$\exp x / c=0.83$

$\exp x / c=1.02$
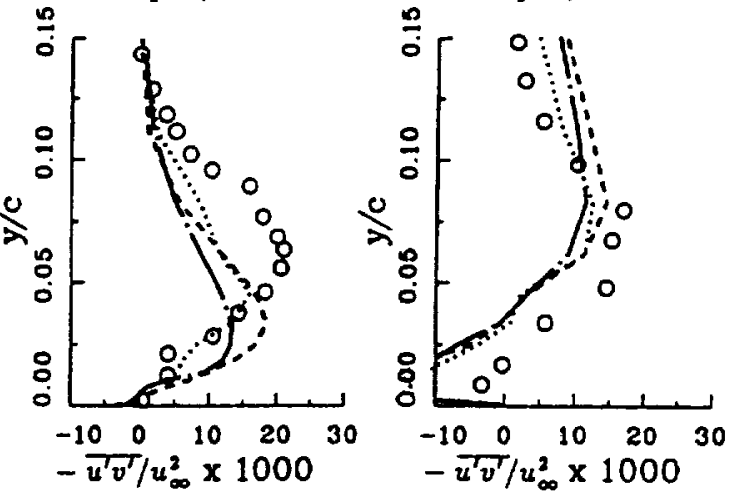

16. Veloctty and Reynolds Stress Proflles for Condilons shown on Figure 15.
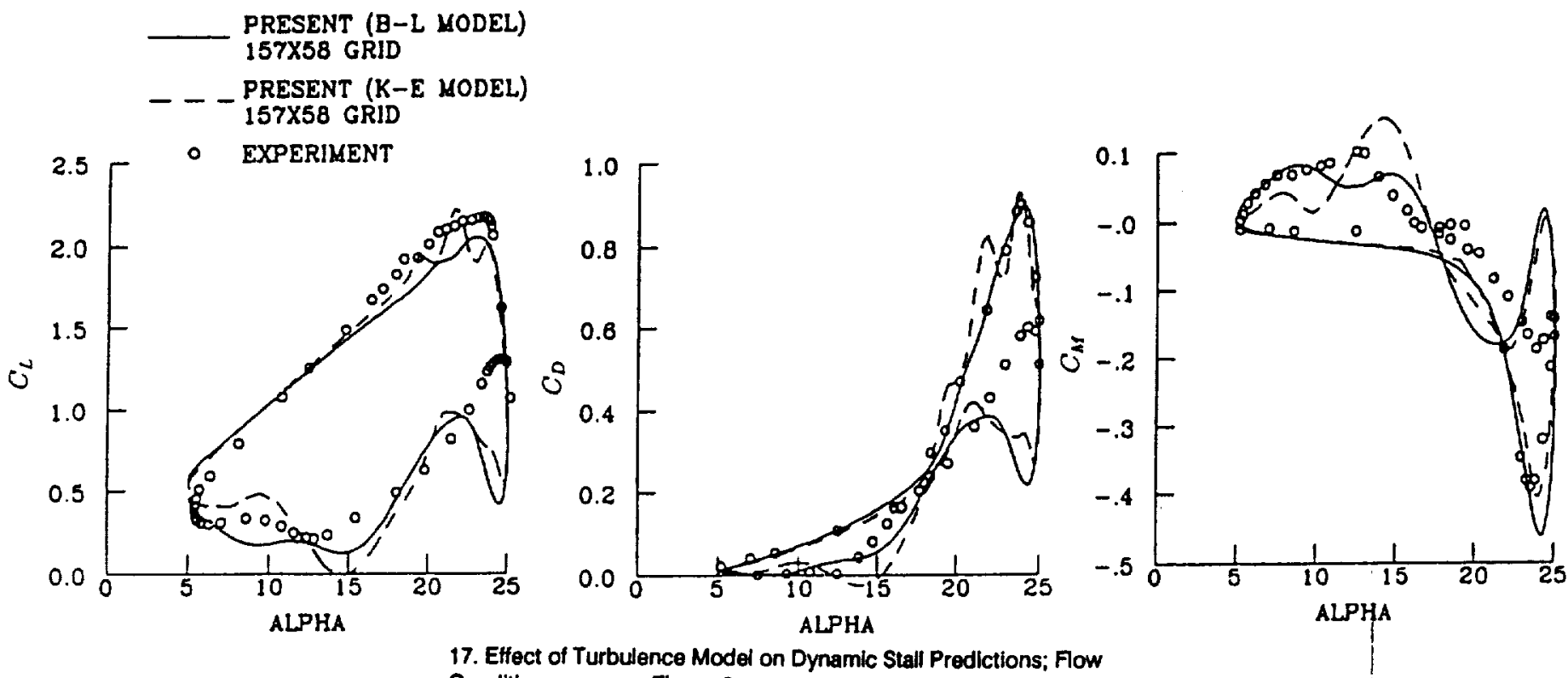

17. Effect of Turbulence Mod
Conditlons same as Figure 6. 


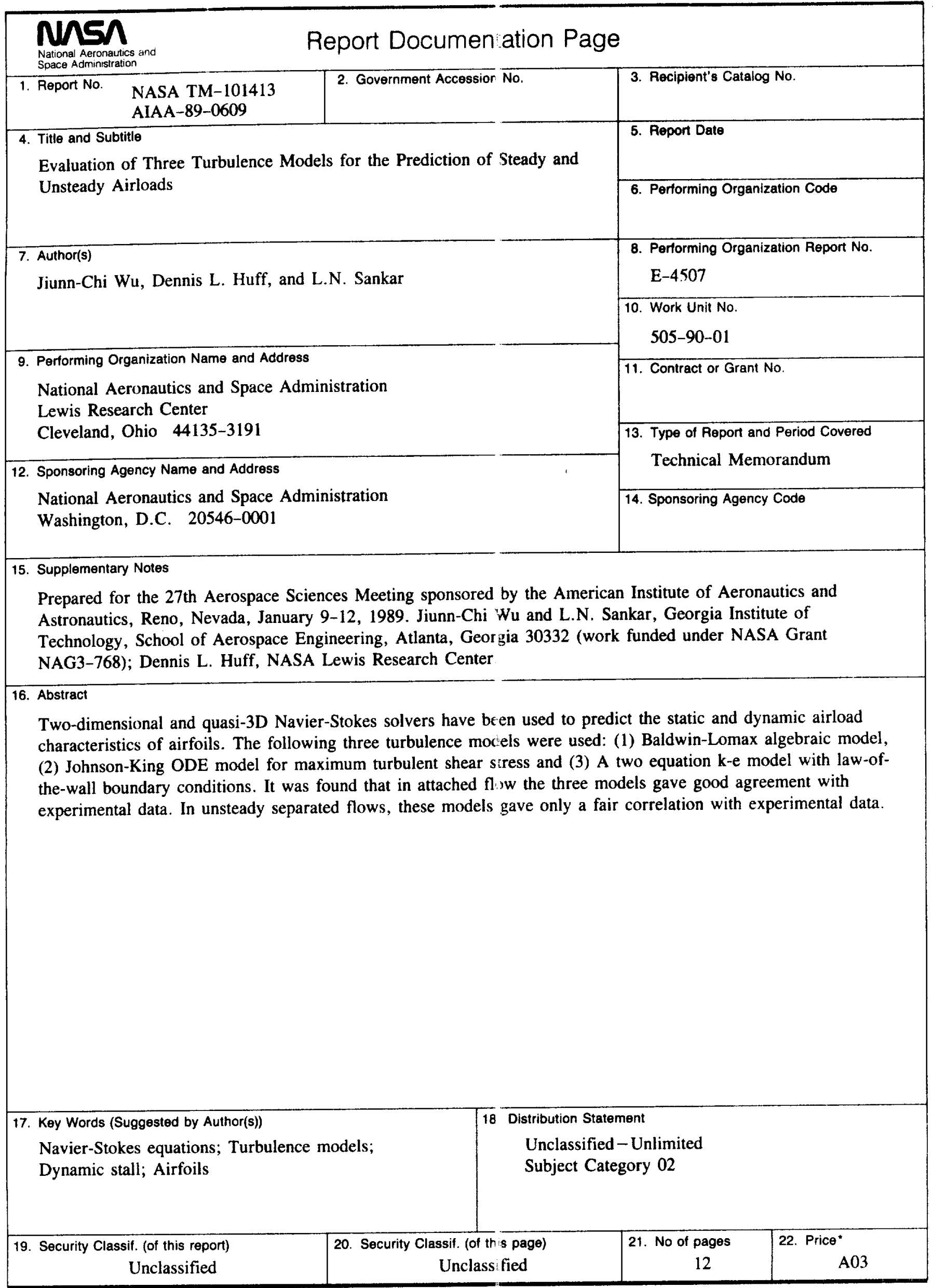


